\title{
Early Onset of Ataxia in Moonwalker Mice Is Accompanied by Complete Ablation of Type II Unipolar Brush Cells and Purkinje Cell Dysfunction
}

\author{
Gabriella Sekerková, ${ }^{1,2 *}$ Jin-Ah Kim, ${ }^{1 *}$ Maximiliano J. Nigro, ${ }^{1}$ Esther B. E. Becker, ${ }^{3}$ Jana Hartmann, ${ }^{4}$ Lutz Birnbaumer, ${ }^{5}$ \\ Enrico Mugnaini, ${ }^{1,2}$ and Marco Martina ${ }^{1}$ \\ Departments of ${ }^{1}$ Physiology and ${ }^{2}$ Cell and Molecular Biology, Northwestern University Feinberg School of Medicine, Chicago, Illinois 60611, ${ }^{3}$ Department \\ of Physiology, Anatomy, and Genetics, MRC Functional Genomics Unit, Oxford OX1 3PT, United Kingdom, ${ }^{4}$ Institute of Neuroscience, Technical University \\ Munich, 80802 Munich, Germany, and 5Laboratory of Neurobiology, National Institute of Environmental Health Sciences, National Institutes of Health, \\ Department of Health and Human Services, Durham, North Carolina 27709
}

\begin{abstract}
Transient receptor potential "canonical" cation channels (TRPC) are involved in many cellular activities, including neuronal synaptic transmission. These channels couple lipid metabolism, calcium homeostasis, and electrophysiological properties as they are calcium permeable and activated through the phospholipase $\mathrm{C}$ pathway and by diacylglycerol. The TRPC3 subunit is abundantly expressed in Purkinje cells (PCs), where it mediates slow metabotropic glutamate receptor-mediated synaptic responses. Recently, it has been shown that heterozygous moonwalker mice, which are a model of cerebellar ataxia, carry a dominant gain-of-function mutation (T635A) in the TRPC3 gene. This mutation leads to PC loss and dysmorphism, which have been suggested to cause the ataxia. However, the ataxic phenotype is present from a very early stage (before weaning), whereas PC loss does not appear until several months of age. Here we show that another class of cerebellar neurons, the type II unipolar brush cells (UBCs), express functional TRPC3 channels; intriguingly, these cells are ablated in moonwalker mice by 1 month of age. Additionally, we show that in moonwalker mice, intrinsic excitability of PCs is altered as early as 3 weeks after birth. We suggest that this altered excitability and the TRPC3-mediated loss of type II UBCs may both contribute to the ataxic phenotype of these mice and that different calcium handling in PCs and type II UBCs may account for the dramatic differences in sensitivity to the moonwalker mutation between these cell types.
\end{abstract}

\section{Introduction}

Cerebellar ataxias represent complex neurological disorders characterized by loss of balance and coordination. All ataxias are characterized by lesions of the cerebellum, but the precise circuitry deficiencies remain mostly obscure. The moonwalker mouse (Becker et al., 2009) is a newly discovered model of cerebellar ataxia in which a gain-of-function mutation (T635A) of the TRPC3 channel leads to Purkinje cell (PC) dysmorphism and some PC loss; the latter becomes progressively evident from 4 months of age in cerebellar hemispheres. The time course of PC loss and the development of ataxia do not overlap, and the phenotype, which is clearly evident at weaning, precedes the PC loss.

Received May 29, 2013; revised 0ct. 18, 2013; accepted Nov. 7, 2013.

Author contributions: G.S., E.M., and M.M. designed research; G.S., J.-A.K., and M.J.N. performed research; E.B.E.B., J.H., and L.B. contributed unpublished reagents/analytic tools; G.S., J.-A.K., M.J.N., E.M., and M.M. analyzed data; G.S., E.M., and M.M. wrote the paper.

This work was supported by NIH Grant NS 09904 (M.M.), DFG Grant SFB 870 (J.H.), and the Intramural Research Program of the NIH (project Z01-ES-101864, L.B.). E.B.E.B. is the recipient of a Research Fellowship from the Royal Society, London. E.B.E.B. thanks Kay E. Davies for generous support.

${ }^{*}$ G.S. and J.-A.K. contributed equally to this work.

The authors declare no competing financial interests.

Correspondence should be addressed to Dr. Marco Martina, Department of Physiology, Northwestern University Feinberg School of Medicine, 303 E. Chicago Avenue, Chicago, IL 60611. E-mail: m-martina@northwestern.edu.

DOI:10.1523/JNEUROSCI.2294-13.2013

Copyright $\odot 2013$ the authors $\quad 0270-6474 / 13 / 3319689-06 \$ 15.00 / 0$
In situ hybridization suggests that the PCs are not the only neurons expressing TRPC 3 in the adult mouse cerebellum (Schilling and Oberdick, 2009). Intense TRPC3 signal is localized to cells of the granular layer in the vestibulocerebellum, particularly the nodulus (lobule X) and uvula (lobule IX). These lobules contain high densities of unipolar brush cells (UBCs), whose distribution suggests they could be the TRPC3-expressing cells. Similar to the granule cells, the UBCs transduce excitatory inputs of a variety of mossy fibers (for review, see Mugnaini et al., 2011) under inhibitory control by the Golgi cells (Dugué et al., 2005; Rousseau et al., 2012); in turn, UBC axons innervate pools of granule cells, ultimately affecting the output of the overlying PCs. Thus, UBCs provide a powerful amplification system for afferent inputs to the cerebellar cortex (Rossi et al., 1995; Diño et al., 2000; Mugnaini et al., 2011). The UBC population is chemically and functionally heterogeneous and consists of two main classes, type I and type II. These populations differ in many aspects, including intrinsic excitability, expression of the calcium-binding protein calretinin (CR), size, and distribution (Nunzi et al., 2002; Kim et al., 2012; Sekerková et al., 2013). Remarkably, one of the distinguishing features between the two cell types is the expression of metabotropic glutamate receptor (mGluR) $1 \alpha$ and its downstream effectors, phospholipase $\mathrm{C} \beta 4$ and diacylglycerol kinase $\beta$, that are found in type II but not type I UBCs (Sekerková et al., 2013). These differences suggest that TRPC3 expression may also 
differ between the two UBC populations as mGluR1 is an activator of transient receptor potential "canonical" cation channels (TRPC) in neurons (Hartmann et al., 2008; Riccio et al., 2009). Indeed, in the cerebellum, TRPC3 was first identified as a postsynaptic channel involved in the slow synaptic transmission mediated by mGluR1 in PCs, and TRPC3-deficient mice lack the mGluR1-dependent slow EPSC and exhibit a distinct walking deficit attributable to a disturbance in cerebellar sensorimotor integration (Hartmann et al., 2008).

Here we report that type II UBCs, but not type I UBCs, express TRPC3 channels, which leads to early and complete loss of these cells in moonwalker mice and may thus contribute to the early onset of the ataxic phenotype.

\section{Materials and Methods}

Animals. This study was performed on 21- to 32-d-old, 4-month-old, and 7-month-old male mice (bred and housed in the vivarium at Northwestern University) and approved by the Northwestern University Center for Comparative Medicine. The following mouse strains were used: CD1 as wild type; $\operatorname{Tg}($ Grp-EGFP)DV197Gsat (Kim et al., 2012); heterozygous moonwalker $\left(M w k l^{+}\right)$(ENU-mutagenized mice; Becker et al., 2009) and their wild-type siblings; and TRPC $3^{-1-}$ (Hartmann et al., 2008).

Histological analysis. Mice were anesthetized with sodium pentobarbi$\operatorname{tal}(60 \mathrm{mg} / \mathrm{kg}$ body weight) and perfused with $4 \%$ formaldehyde in $0.12 \mathrm{M}$ phosphate buffer, $\mathrm{pH}$ 7.4. The brains were either embedded in paraffin or cryoprotected in $30 \%$ sucrose in PBS. Cryoprotected brains were sectioned serially at $25 \mu \mathrm{m}$ in sagittal or coronal planes on a freezing-stage microtome. Fixed brains were paraffin embedded and sectioned by AML Laboratories. The $10-\mu \mathrm{m}$-thick paraffin sections were deparaffinized, rehydrated, and treated with commercial Rodent Decloacker (Biocare Medical) in a pressure cooker to unmask antigen sites.

For bright-field microscopy, sections were incubated with primary antibodies and processed according to an avidin/biotin amplification protocol. For immunofluorescence analysis, sections were incubated with a mixture of primary antibodies, which were visualized by secondary antibodies coupled to Alexa 488 or Alexa 594 (Invitrogen). The following primary antibodies were used: mouse anti-CR (1:2000; Millipore), rabbit anti-CR (1:5000; Swant), mouse anti-mGluR1 $\alpha$ (1:800-1000; BD Pharmingen), rabbit anti-mGluR1 $\alpha$ (1:750; Frontier Institute Company), chicken anti-Tbr2 (1:500; Millipore), rabbit anti-TRPC3 (1:1000, catalog \#OST00301W, Osenses; 1:500, catalog \#ACC-016, Alomone Labs), and rabbit anti-PEP19 (gift from Dr. J. I. Morgan, St. Jude Children's Research Hospital, Memphis, TN). The specificity of the primary antibodies to CR, mGluR1 $\alpha$, PEP19, and Trb2 was validated previously (Mugnaini et al., 1987; Nunzi et al., 2002; Sekerková et al., 2013). The specificity of TRPC 3 antisera was validated by the absence of staining in TRPC $3^{-1-}$ mouse brain sections (Fig. 1I). PC immunoreactivity was apparent in cryosections labeled with the Alomone Labs TRPC3 antibody, whereas UBCs were barely labeled (data not shown). In contrast, the Osenses TRPC3 antibody produced distinct UBC immunolabeling, but only in paraffin sections after antigen retrieval. Notably, PC immunolabeling was attenuated in paraffin sections (Fig. 1A).

Electrophysiological recordings. Mice were deeply anesthetized with isoflurane and decapitated. The cerebellum was removed and placed in ice-cold modified artificial CSF (mACSF; in mM): $125 \mathrm{NaCl}$ ( $87 \mathrm{NaCl}, 75$ sucrose for PCs), $25 \mathrm{NaHCO}_{3}, 2.5 \mathrm{KCl}\left(1.25 \mathrm{KCl}, 1.25 \mathrm{KH}_{2} \mathrm{PO}_{4}\right.$ for PCs), $1.25 \mathrm{NaH}_{2} \mathrm{PO}_{4}, 0.2 \mathrm{CaCl}_{2}, 7 \mathrm{MgCl}_{2}, 25$ glucose (16 glucose for PCs), and 2 kynurenic acid, saturated with $95 \% \mathrm{O}_{2}$ and $5 \% \mathrm{CO}_{2}$. Parasagittal slices, 280-300 $\mu \mathrm{m}$ thick, were cut using a Vibratome (Leica) and incubated in ACSF (in mM): $125 \mathrm{NaCl}, 25 \mathrm{NaHCO}_{3}, 2.5 \mathrm{KCl}\left(1.25 \mathrm{KCl}, 1.25 \mathrm{KH}_{2} \mathrm{PO}_{4}\right.$ for PCs), $1.25 \mathrm{NaH}_{2} \mathrm{PO}_{4}, 1.5 \mathrm{CaCl}_{2}$ (2 $\mathrm{CaCl}_{2}$ for PCs), $1 \mathrm{MgCl}_{2}$, and 25 glucose (10 glucose for PCs), saturated with $95 \% \mathrm{O}_{2}$ and $5 \% \mathrm{CO}_{2}$. Slices were incubated for $20-30 \mathrm{~min}$ at $35^{\circ} \mathrm{C}$ and then at room temperature; they were then transferred to the recording chamber and visualized using infrared differential interference contrast videomicroscopy with waterimmersion, $40 \times$ objective lenses. For current-clamp (CC) recordings,

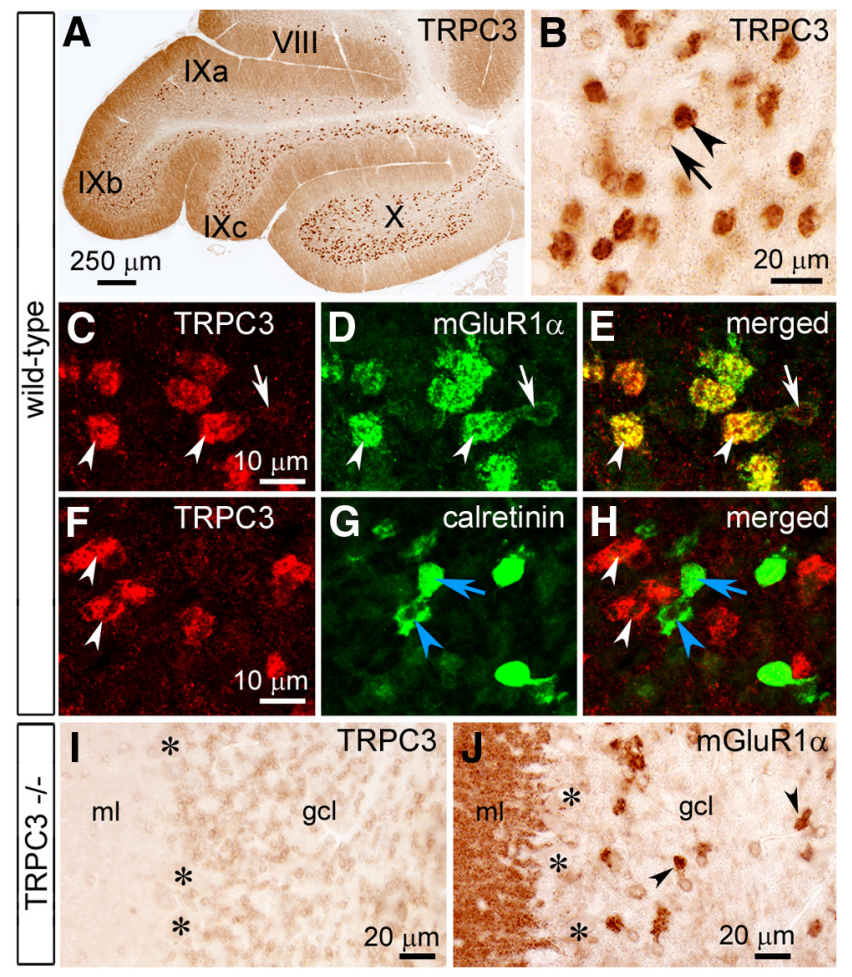

Figure 1. Immunolocalization of TRPC3 in cerebellar paraffin sections from wild-type and $\operatorname{TRPC} 3^{-1-}$ mice. $A$, Enrichment of TRPC3-immunoreactive UBCs in nodulus $(\mathrm{X})$ and ventral uvula (folia IXb and IXC) in a wild-type mouse. Other lobules (IXa, VIII) contain few TRPC3positive UBCs. PCs show only moderate immunostaining. $\boldsymbol{B}$, Immunostained cells in nodulus show typical UBC morphology: mid-sized soma (arrow) and intensely immunoreactive brush (arrowhead). $\mathbf{C} \boldsymbol{H}$, Confocal images from a wild-type mouse illustrate colocalization of TRPC3 with mGluR1 $\alpha$ in type II UBCS $(\boldsymbol{C}-\boldsymbol{E})$. TRPC3 is absent from CR-positive type I UBCs $(\boldsymbol{F}-\boldsymbol{H})$. Arrows and arrowheads indicate somata and dendritic brushes, respectively (white, type II UBC; blue, type I UBCS). I, J, TRPC3 immunostaining is abolished in the cerebellar sections from a $\operatorname{TRPC} 3^{-/-}$mouse (I); however, the immunostaining of mGluR1 $\alpha$ in type II UBCs (arrowheads) and $\mathrm{PCs}$ (labeling in $\mathrm{ml}$ ) appears unaffected $(\boldsymbol{J})$. Asterisks indicate PC somata. ml, Molecular layer; gcl granule cell layer.

slices were superfused with ACSF. For voltage-clamp (VC) recording of TRP current in UBCs, slices were superfused with mACSF in which $\mathrm{CaCl}_{2}$ was substituted by $\mathrm{BaCl}_{2}(1 \mathrm{~mm})$ and contained $5 \mathrm{~mm}$ TEA, $1 \mathrm{~mm}$ $\mathrm{CsCl}$, and $10 \mu \mathrm{M}$ nimodipine. $\mathrm{VC}$ and $\mathrm{CC}$ recordings were performed in the presence of $2 \mathrm{~mm}$ kynurenic acid, $50 \mu \mathrm{M}$ picrotoxin, and $1 \mu \mathrm{M}$ strychnine to block fast synaptic transmission. All UBC recordings were performed from nodulus at $32-34^{\circ} \mathrm{C}$. EGFP-tagged type II UBCs were identified using epifluorescence illumination in $\mathrm{Tg}$ (Grp-EGFP) DV197Gsat mice. Patched UBCs were filled with Alexa $405(100 \mu \mathrm{M})$ for post hoc cell-type identification. UBC types were identified by double immunostaining with antibodies against mGluR $1 \alpha$ and CR.

Recording pipettes were pulled from borosilicate glass (Sutter Instruments) and had resistances of 5-8 M $\Omega$ (for UBCs) and 4-6 (for PCs) when filled with an internal solution (in $\mathrm{mM}$ ): $120 \mathrm{~K}$-gluconate, $20 \mathrm{KCl}$, 10 HEPES, $10 \mathrm{Na}_{2}$-phosphocreatine, $4 \mathrm{Mg}$-ATP, $0.3 \mathrm{Na}$-GTP for PCs; $138 \mathrm{CsCl}, 2 \mathrm{NaCl}, 2 \mathrm{MgCl}_{2}, 0.1$ EGTA, $2 \mathrm{Na}_{2} \mathrm{ATP}, 0.1 \mathrm{NaGTP}, 10$ HEPES, and 10 phosphocreatine di(tris) ( $\mathrm{pH} 7.3$ with $\mathrm{KOH})$. A total of $5 \mathrm{mM}$ QX-314 was added to block voltage-gated $\mathrm{Na}^{+}$and $\mathrm{K}^{+}$channels. Recordings were performed with Axopatch 200B amplifiers (Molecular Devices). Current signals were low-pass filtered at 2 or $5 \mathrm{kHz}$ and digitized (10-20 kHz) using either Digidata 1321A or 1200, controlled by pClamp software (Molecular Devices). Signals from CC recordings were sampled at $20 \mathrm{kHz}$ and filtered at $10 \mathrm{kHz}$. All the VC recordings had series resistances of $<50 \mathrm{M} \Omega$ that were compensated $40-60 \%$. All chemicals were from Sigma-Aldrich, except picrotoxin, (RS)-3,5-dihydroxyphenylglycine (DHPG), and 1-[4-[(2,3,3-trichloro-1-oxo-2-prop-en-1-yl)amino] phenyl]-5-(trifluoromethyl)-1H-pyraz-ole-4-carboxylic acid (Pyr3), 

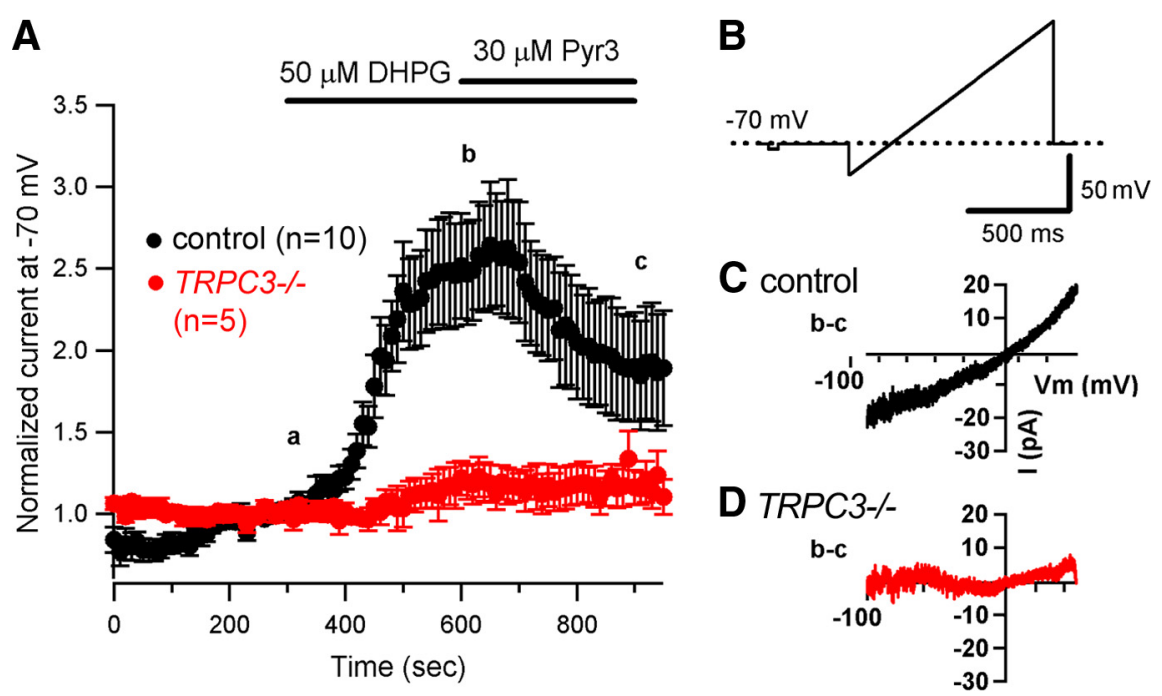

Figure 2. TRPC3 channels expressed in type II UBCs are functional. $\boldsymbol{A}$, Time course of the current (at $-70 \mathrm{mV}$ ) in control conditions and in the presence of DHPG and DHPG + Pyr3 in type II UBCs obtained from Tg(Grp-EGFP)DV197Gsat (control, black symbols, $n=10$ ) and $\operatorname{TRPC} 3^{-1-}$ (red symbols, $n=5$ ) mice. Currents were normalized to the control current (averaged between 240 and $300 \mathrm{~s}$ ). A total of $50 \mu \mathrm{m} \mathrm{DHPG} \mathrm{increased} \mathrm{the} \mathrm{current} \mathrm{up} \mathrm{to} 2.5$ fold in UBCs from control mice. Subsequent application of Pyr3 $(30 \mu \mathrm{M})$, a TRPC3 antagonist, decreased the DHPG-sensitive currents by $54.5 \pm 10.6 \%(p<0.05$, ANOVA with Newman-Keuls post hoc test). In type II UBCs of TRPC ${ }^{-1-}$ mice, DHPG only induced a detectable current in three of five cells, and it was not blocked by Pyr3. B, Voltage ramp used to measure the Pyr3-sensitive current shown in $\mathbf{C}$ and $\boldsymbol{D}$. C, $\boldsymbol{D}$, Representative Pyr3-sensitive currents obtained by digital subtraction of the average current in DHPG and Pyr 3 from the average current in DHPG (marked as c and $\mathrm{b}$ in $\boldsymbol{A}$, respectively) in control mice $(\boldsymbol{C})$ and $\operatorname{TRPC} 3^{-1-}$ mice $(\boldsymbol{D})$.

which were from Abcam. Data are presented as mean \pm SEM; error bars in the figures also represent SEM.

\section{Results}

TRPC3 is selectively expressed in type II UBCs

We aimed to identify the TRPC3-expressing cells in the granule cell layers previously observed by Schilling and Oberdick (2009). To this end, we used the TRPC3 antibody from Osenses, which showed distinct TRPC3 labeling of UBCs in the paraffin sections (Fig. 1). The staining revealed a moderate immunolabeling in the UBC soma and intense immunolabeling in the dendritic brush (Fig. 1B). UBC axons and axonal terminals (intrinsic mossy fibers) were immunonegative. With the exception of PCs, whose immunolabeling was attenuated in the paraffin sections (Fig. $1 A)$, no other cerebellar neurons were labeled.

TRPC3-labeled UBCs showed typical distribution, with enrichment in the vestibulocerebellum, especially in the nodulus, in folia IXb and IXc of uvula (Fig. 1A), and in the flocculus/parfloccular transition zone (data not shown). Moreover, TRPC3labeled UBCs were scattered throughout other cerebellar lobes, suggesting that TRPC3 labeling was mostly, if not entirely, limited to type II UBCs. To unequivocally identify the TRPC3expressing UBC subtype, we used confocal imaging and double labeling with subtype-specific UBC markers CR (type I) and mGluR $1 \alpha$ (type II). These experiments revealed colabeling of mGluR $1 \alpha$ and TRPC3 in type II UBCs (Fig. $1 C-E$ ). In contrast, no type I UBC showed TRPC3 labeling (Fig. $1 F-H$ ). As our data indicate type II UBCs as the only other source of TRPC3 besides PCs in the cerebellum, we focused our further investigation on these cell types.

First, we looked at type II UBCs in TRPC $3^{-1-}$ mice. As expected, no TRPC3 staining was detectable (Fig. 1I) although both type II UBCs and PCs were normally present in the cerebellum and contained mGluR1 $\alpha$ (Fig. 1J; see also Hartmann et al., 2008).
The morphology and cerebellar distribution of type II UBCs were similar to wildtype mice and were not investigated further.

Next, we performed patch-clamp recordings from acute slices to examine whether TRPC3 channels in type II UBCs are functional. For these experiments, we used $\operatorname{Tg}$ (Grp-EGFP)DV197Gsat mice, in which EGFP is expressed in type II (mGluR $1 \alpha$-positive) UBCs and allows their identification (Kim et al., 2012). In PCs, TRPC3 current is mediated by mGluR1 activation (Hartmann et al., 2008; Becker et al., 2009). Thus, we tested the mGluR1 agonist DHPG $(50 \mu \mathrm{M})$ on type II UBCs. UBCs were held at $-70 \mathrm{mV}$ and probed with voltage ramps $(-100$ to $50 \mathrm{mV}, 1 \mathrm{~s}$, at $0.1 \mathrm{~Hz}$; Fig. $2 B$ ). Bath application of DHPG caused an $\sim 2.5$-fold current increase (Fig. 2A). mGluR1 activation by DHPG, however, may activate other channels beside TRPC3; thus, DHPG activation of TRPC3 current was verified by applying the TRPC3 blocker Pyr3 (Kiyonaka et al., 2009) on top of DHPG. Pyr3 (30 $\mu \mathrm{M})$ application led to a significant reduction in the DHPGactivated current (Pyr3 reduced the DHPGactivated current by $54.5 \pm 10.6 \%, p<$ $0.05, n=10$; Fig. $2 A$ ). Off-line subtraction allowed the isolation of the DHPG-activated, Pyr3-sensitive TRPC3 current (Fig. 2C). No Pyr3-sensitive current was detected in TRPC $3^{-1-}$ mice (Fig. $2 D$ ), although DHPG still evoked a small current in three of five cells (Fig. $2 A$, red trace). Together, these data show that activation of group I mGluR gates TRPC3 channels in type II UBCs.

\section{Loss of type II UBCs in the moonwalker mouse}

From 3 weeks of age, $\mathrm{Mwk} /{ }^{+}$mice are severely ataxic, but a PCs loss is evident only from 4 months of age (Becker et al., 2009). Our data show that, similar to PCs, type II UBCs express functional TRPC3 channels, and we hypothesized that the moonwalker mutation would similarly affect both cell types. To our surprise, however, neither TRPC3 nor mGluR1 $\alpha$ immunolabeling detected any type II UBC in the cerebella of 4-month-old $M w k /^{+}$mice (data not shown). Subsequently, we analyzed sections from 1-month-old $\mathrm{Mwk} /{ }^{+}$mice and found that type II UBCs were almost completely absent (Fig. $3 A, B, E, F, I$ ). mGluR $1 \alpha$-immunolabeled UBCs were extremely rare in the vestibulocerebellum (Fig. $3 E, F, I)$ and absent in other lobules. Similar to wild-type cerebella, CR-positive type I UBCs in $\mathrm{Mwk} /{ }^{+}$cerebella were restricted to the vestibulocerebellum (Fig. 3C,G). Unlike mGluR1 $\alpha$-positive UBCs, which completely disappear after 1 month of age, CR-positive UBCs were retained in older animals, albeit in reduced numbers (Fig. $3 I, J)$. To verify that the absence of $\mathrm{mGluR} 1 \alpha \mathrm{UBC}$ is caused by cell loss and not to changes in mGluR $1 \alpha$ expression, we used immunolabeling to detect the presence of Tbr2 (UBC-specific marker; Englund et al., 2006; Sekerková et al., 2013) in UBC nuclei. Tbr2immunoreactive nuclei were present in the granule cell layer of the vestibulocerebellum of both wild-type (Fig. 3D) and $\mathrm{Mwk} /^{+}$ (Fig. $3 \mathrm{H}$ ) mice. Notably, in $M w k /^{+}$mice, the density of Tbr2positive nuclei was reduced, and their distribution overlapped with type I UBCs (Fig. 3I,J). 

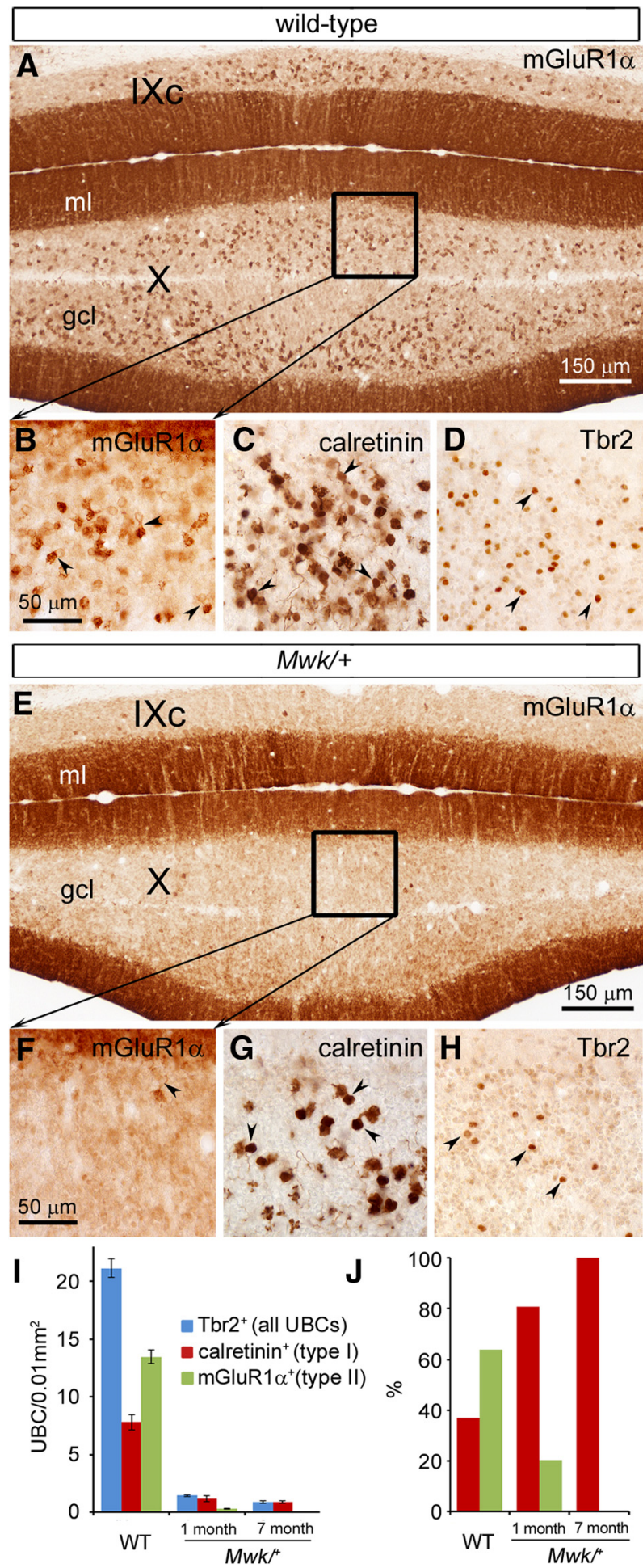

Figure 3. UBC-specific immunostaining in cerebellar cryosections of 1-month-old wild-type and moonwalker mice. $\boldsymbol{A}-\boldsymbol{D}, \mathrm{mGluR1} \alpha(\boldsymbol{A}, \boldsymbol{B}), \mathrm{CR}(\boldsymbol{C})$, and Tbr2 (D) immunolabeling in the nodulus $(X)$ and folium IX of wild-type mice. $A-C$, In the molecular layer $(\mathrm{ml})$, the $P($ dendritic arbors are mGluR1 $\alpha$ positive $(\boldsymbol{A})$. In the granule cell layer ( $\mathrm{gcl})$, type I $(\boldsymbol{C})$ and type II $(\boldsymbol{A}, \boldsymbol{B})$ UBCs contain CR and $m G l u R 1 \alpha$, respectively. D, Tbr2 labeling of UBC nuclei. $\boldsymbol{E}-\boldsymbol{G}, \ln M w k /^{+}$mice, the mGluR1 $\alpha$-positive type II UBCs are depleted $(\boldsymbol{E}, \boldsymbol{F})$, whereas some (R-positive type I UBCs are still present (G). The $\mathrm{ml}$ shows distinct mGluR1 $\alpha$ immunolabeling $(\boldsymbol{E})$. $\boldsymbol{H}$, Density of Tbr2positive nuclei is reduced in $M w k /{ }^{+}$mice. $\boldsymbol{B}-\boldsymbol{D}$ and $\boldsymbol{F}-\boldsymbol{H}$ were taken at the same magnifications from adjacent sections. Arrowheads indicate UBCs. I, J, Densities of UBCs in wild-type (WT; 1.5-month-old) mice and 1-and 7-month-old $M w k /^{+}$mice. The density of Tbr2-positive nuclei was reduced by $\sim 93 \%$. In $J$, type I and type II UBCs are shown as fractions of all UBCs, determined by counting the Tbr2-positive nuclei.

\section{PCs of moonwalker mice are functionally altered by 3 weeks after birth}

Our data show that in $\mathrm{Mwk}^{+}$mice, type II cerebellar UBCs are ablated much earlier than PCs, whose number is still normal several months after the appearance of the ataxic symptoms. This suggests that UBC ablation may be critical for the ataxic phenotype.

However, the presence of PCs with relatively normal morphology (Fig. 4A-C) does not imply that these cells are functionally intact (Becker et al., 2009). Therefore, we performed patch-clamp recordings in slices from 21- to 28-d-old wild-type and $\mathrm{Mwk} /^{+}$mice and compared the intrinsic electrophysiological properties of PCs. Cell-attached recordings showed that although all $(n=8)$ PCs recorded in wild-type animals were spontaneously firing, only $\sim 10 \%$ (3 of 30) of the PCs of $M w k /^{+}$mice were firing (Fig. $4 D, E, J)$. This could be attributable to either hyperpolarization or depolarization block. Whole-cell recordings showed that, similar to the extracellular measurements, all wild-type PCs were firing (Fig. $4 F$ ), whereas $50 \%$ of the PCs from $M w k /^{+}$mice were silent and depolarized $\left(V_{\mathrm{m}}=-33 \pm 1.2 \mathrm{mV}, n=7\right.$, data not shown). Interestingly, the $M w k{ }^{+}$PCs that were active (Fig. 4G), fired at slightly higher frequency than PCs from wild-type $(83 \pm 23 \mathrm{~Hz}$ vs $69 \pm 11 \mathrm{~Hz}, n=7$ and 8 , respectively). These data suggest that $\mathrm{Mwk}^{+}$PCs are generally depolarized and that the silent PCs observed in cell attached recordings were in a depolarization block. In keeping with this interpretation, when silent $M w k /^{+}$ PCs were hyperpolarized with injection of negative current, they were able to fire, although at a slower frequency than control cells (Fig. $4 H, I$ ). Thus, PCs of $M w k /{ }^{+}$mice show important changes in intrinsic electrophysiological properties early in postnatal development.

\section{Discussion}

Here we report several novel and intriguing findings. We show that TRPC 3 is differentially expressed in the two UBC populations; as a consequence of TRPC3 expression in type II UBCs, these neurons are ablated in moonwalker mice, and the time course of UBC cell loss better matches the ataxic phenotype than the PC loss, which is incomplete (most cells are spared) and only appears much later. Interestingly, the density of type I UBCs, which do not express TRPC 3 in adult mice, is also severely reduced; whether this is caused by transient TRPC 3 expression in some developmental stage or attributable to some deafferentation remains to be established. We also show that the intrinsic electrophysiological properties of PCs are already compromised at an early stage; each of these events may possibly play a causal role in establishing the ataxic phenotype. Finally, we show that the same TRPC 3 mutation has dramatically different effects in UBCs, which are eliminated by 1 month, and PCs, which do not show cell death for several months.

\section{TRPC3 expression in UBCs and ataxia}

We demonstrated TRPC3 protein expression in type II UBCs. Electrophysiological recordings from these cells revealed that mGluR1 activation elicits a current sensitive to the TRPC3 blocker Pyr3, supporting the idea that functional TRPC3 channels are inserted into the membrane of these interneurons. In contrast, TRPC3 immunoreactivity was absent in type I UBCs, providing further evidence of diversification within the UBC population. Type II UBCs are virtually absent in moonwalker mice as early as 1 month after birth, an age when the ataxic phenotype is already established and PCs show only minor morphological alterations. Whether type II UBCs develop normally 


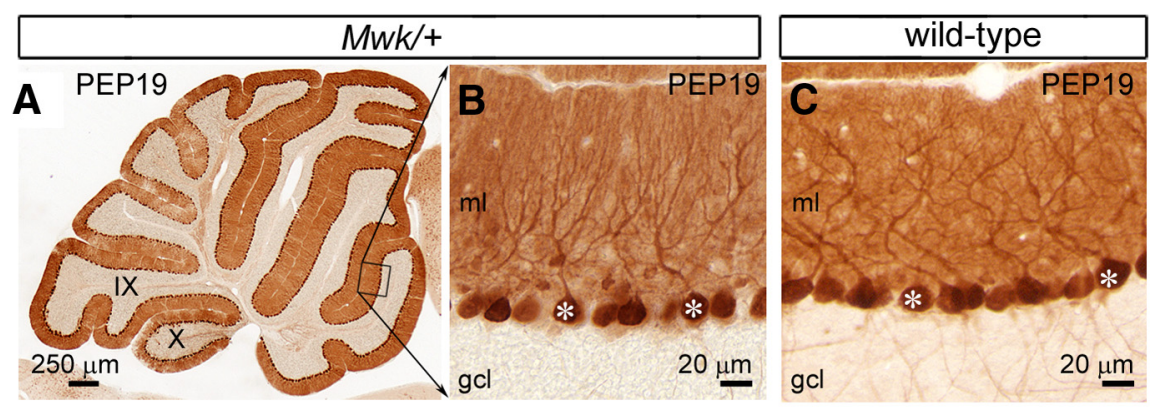

D
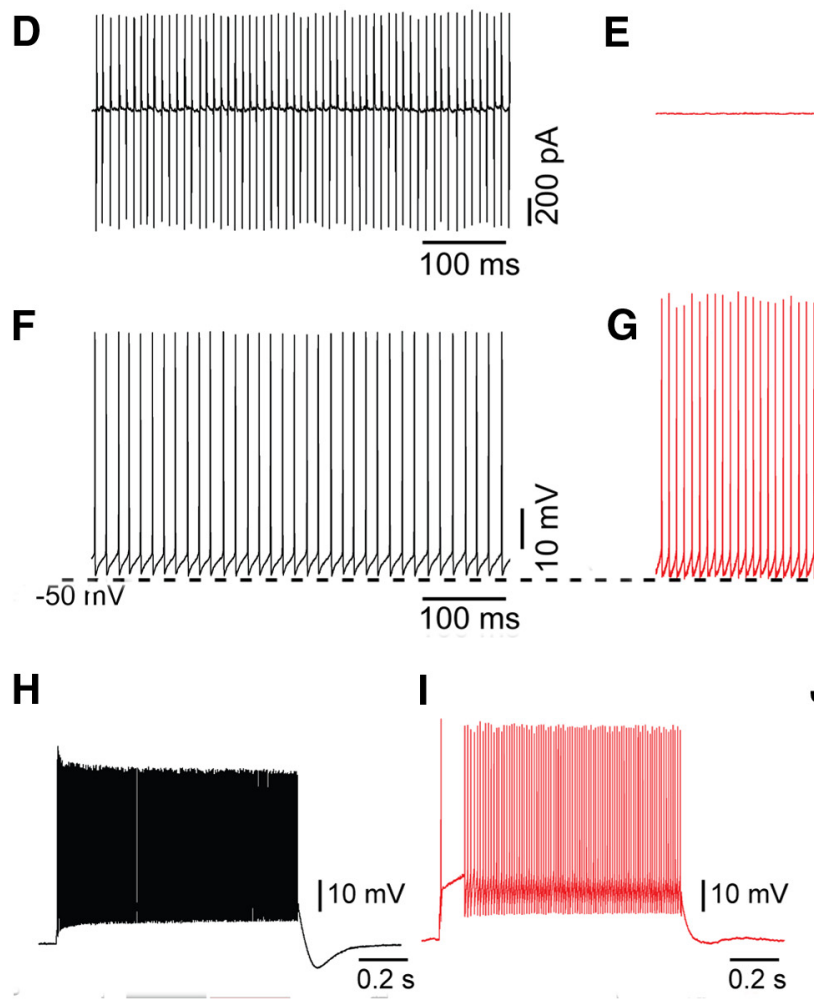

E

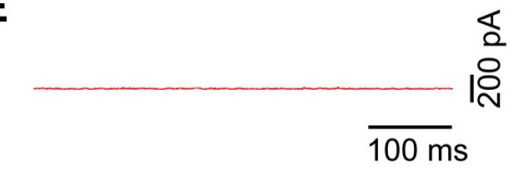

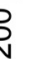
resent a model for spinocerebellar ataxia 14 , in which a mutation in PKC $\gamma$ results in insufficient phosphorylation of an inhibitory site of the TRPC3 channel (Adachi et al., 2008).

\section{Differences in calcium homeostasis may explain the different effect of the moonwalker mutation in PCs and UBCs}

In the PCs, which abundantly express TRPC3 throughout their somatodendritic compartments, the moonwalker mutation results in dysmorphic dendritic arbors, with a moderate cell loss apparent at 4 months of age. Thus, we expected that the brush dendrioles of type II UBCs of Mwk $/{ }^{+}$mice would also be reduced in length, size, or number. Yet, the early ablation of the type II UBC subclass was surprising. Because TRPC3 channels are highly calcium permeable, it is tempting to propose that the moonwalker gain-offunction mutation produces increased calcium influx that eventually overcomes the cell's handling capability and leads to cell death. Because PCs show intense expression of calcium-binding and calcium-regulating proteins (calbindin, parvalbumin, calmodulin, CaMKII), whereas no major calciumbinding protein is strongly expressed by type II UBCs (Nunzi et al., 2002; Kim et al., 2012), the calcium buffering capacity ing of PCs in a sagittal cerebellar cryosection from 1-month-old $\mathrm{Mwk}^{+}$and wild-type mice. At higher magnification, the morphology of $M w k /^{+} \mathrm{PCs}(\boldsymbol{B}$, asterisks) resembles that of wild-type PCs (C). ml, molecular layer; gcl, granule cell layer. $\boldsymbol{D}, \boldsymbol{E}$, Cell-attached recordings show spontaneous firing in $\mathrm{PCs}$ from wild-type mice but no spontaneous activity in $M w k /{ }^{+} \mathrm{PCs} . \boldsymbol{F}, \boldsymbol{G}$, Whole-cell recordings of spontaneous firing of PCs from wild-type and $M w k /^{+}$mice. The dashed line represents $-50 \mathrm{mV}$. $\boldsymbol{H}, \boldsymbol{I}$, Voltage responses to depolarizing current injections (1.3 nA; cells held at $-70 \mathrm{mV}$ with bias current injection) in PCs from wild-type and $\mathrm{Mwk}^{+}$mice. J, Fraction of wild-type (WT) and $\mathrm{Mwk} /{ }^{+} \mathrm{PCs}$ that were intrinsically firing in cell-attached recordings.

and then die early or are not properly generated will require additional studies, but the fact that some, although very few, type II UBCs are still detectable in 1-month-old $M w k /{ }^{+}$mice suggests that they develop normally. $M w k / M w k$ mutants are not viable; on the other hand, $M w k /^{+}$mice, although half the size of their wildtype littermates, are fertile but display gait abnormalities on completion of the postnatal cerebellar morphogenesis (postnatal day 21) (Becker et al., 2009). The ataxic phenotype of moonwalker mice was attributed to impaired development of the PC dendrites, with a moderate PC loss developing over time between 4 and 9 months of age. Because massive ablation of type II UBCs is already present in 1-month-old $M w k /^{+}$mice, it is possible that their disappearance contributes to the ataxic phenotype, although the PC electrophysiological dysfunction is also likely to contribute (Shakkottai et al., 2011). Interestingly, unlike type I UBCs, type II UBCs are also present in spinocerebellar lobules, supporting the idea that these neurons may contrib- might greatly differ in the two cell types. Moreover, cytoplasmic volume is very small in UBCs compared with PCs. A combination of these factors might explain why the same mutation produces much earlier cell death of type II UBCs compared with PCs.

\section{References}

Adachi N, Kobayashi T, Takahashi H, Kawasaki T, Shirai Y, Ueyama T, Matsuda T, Seki T, Sakai N, Saito N (2008) Enzymological analysis of mutant protein kinase Cgamma causing spinocerebellar ataxia type 14 and dysfunction in Ca2+ homeostasis. J Biol Chem 283:19854-19863. CrossRef Medline

Becker EB, Oliver PL, Glitsch MD, Banks GT, Achilli F, Hardy A, Nolan PM, Fisher EM, Davies KE (2009) A point mutation in TRPC3 causes abnormal Purkinje cell development and cerebellar ataxia in moonwalker mice. Proc Natl Acad Sci U S A 106:6706-6711. CrossRef Medline

Diño MR, Schuerger RJ, Liu Y, Slater NT, Mugnaini E (2000) Unipolar brush cell: a potential feedforward excitatory interneuron of the cerebellum. Neuroscience 98:625-636. CrossRef Medline

Dugué GP, Dumoulin A, Triller A, Dieudonné S (2005) Target-dependent 
use of co-released inhibitory transmitters at central synapses. J Neurosci 25:6490-6498. CrossRef Medline

Englund C, Kowalczyk T, Daza RA, Dagan A, Lau C, Rose MF, Hevner RF (2006) Unipolar brush cells of the cerebellum are produced in the rhombic lip and migrate through developing white matter. J Neurosci 26:91849195. CrossRef Medline

Hartmann J, Dragicevic E, Adelsberger H, Henning HA, Sumser M, Abramowitz J, Blum R, Dietrich A, Freichel M, Flockerzi V, Birnbaumer L, Konnerth A (2008) TRPC3 channels are required for synaptic transmission and motor coordination. Neuron 59:392-398. CrossRef Medline

Kim JA, Sekerková G, Mugnaini E, Martina M (2012) Electrophysiological, morphological and topological properties of two histochemically distinct subpopulations of cerebellar unipolar brush cells. Cerebellum 11:10121025. CrossRef Medline

Kiyonaka S, Kato K, Nishida M, Mio K, Numaga T, Sawaguchi Y, Yoshida T, Wakamori M, Mori E, Numata T, Ishii M, Takemoto H, Ojida A, Watanabe K, Uemura A, Kurose H, Morii T, Kobayashi T, Sato Y, Sato C, et al. (2009) Selective and direct inhibition of TRPC3 channels underlies biological activities of a pyrazole compound. Proc Natl Acad Sci U S A 106:5400-5405. CrossRef Medline

Martin-Trujillo A, Iglesias-Platas I, Coto E, Corral-Juan M, San Nicolás H, Corral J, Volpini V, Matilla-Dueñas A, Monk D (2011) Genotype of an individual single nucleotide polymorphism regulates DNA methylation at the TRPC3 alternative promoter. Epigenetics 6:1236-1241. CrossRef Medline

Mugnaini E, Berrebi AS, Dahl AL, Morgan JI (1987) The polypeptide PEP-19 as a marker for Purkinje neurons in cerebellar cortex and cartwheel neurons in the dorsal cochlear nucleus. Arch Ital Biol 126:41-67. Medline

Mugnaini E, Sekerková G, Martina M (2011) The unipolar brush cell: a remarkable neuron finally receiving deserved attention. Brain Res Rev 66:220-245. CrossRef Medline

Nunzi MG, Shigemoto R, Mugnaini E (2002) Differential expression of calretinin and metabotropic glutamate receptor mGluRlalpha defines subsets of unipolar brush cells in mouse cerebellum. J Comp Neurol 451: 189-199. CrossRef Medline

Riccio A, Li Y, Moon J, Kim KS, Smith KS, Rudolph U, Gapon S, Yao GL, Tsvetkov E, Rodig SJ, Van't Veer A, Meloni EG, Carlezon WA Jr, Bolshakov VY, Clapham DE (2009) Essential role for TRPC5 in amygdala function and fear-related behavior. Cell 137:761-772. CrossRef Medline

Rossi DJ, Alford S, Mugnaini E, Slater NT (1995) Properties of transmission at a giant glutamatergic synapse in cerebellum: the mossy fiber-unipolar brush cell synapse. J Neurophysiol 74:24-42. Medline

Rousseau CV, Dugué GP, Dumoulin A, Mugnaini E, Dieudonné S, Diana MA (2012) Mixed inhibitory synaptic balance correlates with glutamatergic synaptic phenotype in cerebellar unipolar brush cells. J Neurosci 32:46324644. CrossRef Medline

Schilling K, Oberdick J (2009) The treasury of the commons: making use of public gene expression resources to better characterize the molecular diversity of inhibitory interneurons in the cerebellar cortex. Cerebellum 8:477-489. CrossRef Medline

Sekerková G, Watanabe M, Martina M, Mugnaini E (2013) Differential distribution of phospholipase $\mathrm{C}$ beta isoforms and diaglycerol kinase-beta in rodents cerebella corroborates the division of unipolar brush cells into two major subtypes. Brain Struct Funct. Advance online publication. Retrieved Nov. 20, 2013. doi:10.1007/s00429-013-0531-9. CrossRef Medline

Shakkottai VG, do Carmo Costa M, Dell'Orco JM, Sankaranarayanan A, Wulff H, Paulson HL (2011) Early changes in cerebellar physiology accompany motor dysfunction in the polyglutamine disease spinocerebellar ataxia type 3. J Neurosci 31:13002-13014. CrossRef Medline 